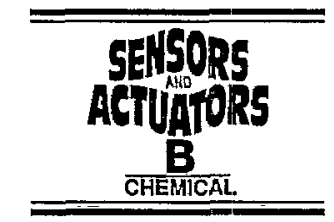

\title{
Waveguide coupling gratings for high-sensitivity biochemical sensors
}

\author{
V.A. Sychugov ${ }^{a}$, A.V. Tishchenko ${ }^{a}$, N.M. Lyndin ${ }^{a}$, O. Parriaux ${ }^{b, c, *}$ \\ a Institute of General Physics, Vavilov Street 38, Moscow 117942, Russia \\ ${ }^{\circ}$ University of Twente, MESA Research Institute, PO Box 217, NL-7500 AE Enschede, Netherlands \\ - Saint-Etienne University, TSI Laboratory, 42023 Saint-Etienne, France
}

\begin{abstract}
Grating coupling is now currently used in evanescent-wave biochemical sensors as a waveguide coupling element or as the sensing element. In most coupling cases of practical interest, the Rayleigh-Fourier method is valid, and leads to physically meaningful analytical solutions allowing grating coupling to be designed in simple terms. In the present paper the emphasis is placed on the grating as a waveguide coupling element.

Keywords: Biochenicical sensors; Waveguide coupling gratings
\end{abstract}

\section{Introduction}

The possibility of measuring biological and chemical reactions taking place at the corrugated surface of an optical waveguide was first demonstrated in 1983 [1]. This possibility relies on the change of the effective index of a waveguide mode due to the change of the refractive index of a layer adjacent to the waveguide where the reaction occurs. Since then, many variations of the original concept have been designed and demonstrated. One has given rise to practical applications in the form of an instrument performing the measurement of a biological reaction at the surface of a waveguide grating [2].

However, it is likely that such an approach will soon be applied more widely and systematically in the form of lowcost sensors in pharmaceutical drug screening, in environment monitoring, in food control and in the diagnosis of diseases. At this stage, the designer will have to perform the optimization of his optical transducer structures according to a list of specifications and constraints. It is quite straightforward to find out the waveguide parameters for a maximum sensing sensitivity [3], but it is less straightforward to define the waveguide grating configuration and parameters achieving a given system functionality in an optimized fashion. The authors of the present paper have provided during the last two decades most of the concepts, results and tools for performing this task. These are exhaustively referred to in Ref. [4]. The usefulness and rich physical content of the present

\footnotetext{
* Corresponding author. Fax: +31534893343 or +33477421820 .
}

approach are confirmed by the renewed interest in the very recent literature [5], where the same problem and method are taken up anew. The authors are discussing here the basic elements that are specific for this field of applications and will help designers to optimize their waveguide grating sensor systems.

\section{The important specific features of coupling gratings}

The role that is usually assigned to the waveguide grating in evanescent-wave biosensors is first of all to perform the coupling between a free-space wave and a sensing guided mode. In general, a first grating couples an incident Gaussian beam from the substrate side into a guided mode, and a second grating couples out the guided wave back into the substrate after its evanescent-field tail has sensed the waveguide overlay. Many variations are possible whereby the grating performs the coupling function only, the sensing being achieved along the non-corrugated waveguide section [6].

The waveguide grating can also be directly assigned the sensing function in cases where the biochemical reaction takes place in the grating area. The readout of the measurand is made by the angle under which the synchronism condition between the free-space and the guided waves is satisfied or, for a given incidence angle, by the grating period satisfying the synchronism [7].

In achieving one or both functions described above, the grating coupler therefore appears as the key functional element of an evanescent-wave biochemical sensor. Fig. 1 illus- 


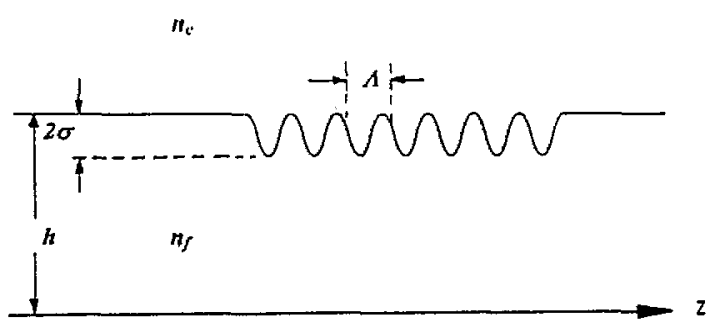

$n$

Fig. 1. Sketch of the typical waveguide grating sensor considered here with the corrugation at the guide-cover interface,

trates the basic structure considered in the present application. It represents a step-index waveguide of thickness $h$, refractive index $n_{\mathrm{f}}$, deposited on a semi-infinite substrate of refractive index $n_{\mathrm{s}}$. The cover is a material of index $n_{\mathrm{c}}$, usually a fluid. The grating is a periodic corrugation of period $\Lambda$, with peakto-peak amplitude $2 \sigma$. The incidence usually takes place from the substrate side under an angle $\theta$. The grating can either be made at the guide-cover interface, as in the case of embossed sol-gel films [8], or it can also be a double undulation first defined at the substrate surface with amplitude $\sigma_{s}$, as in the case of hot-embossed substrates [7,9]. After the waveguide deposition, an undulation of amplitude $\sigma_{\mathrm{c}}$ remains at the guide-cover interface.

The first and most important feature of a coupling grating is its radiation coefficient, $\alpha_{\text {rad }}$. Assuming a propagating mode of electric-field amplitude $E_{0}$ before reaching a grating section, $E=E_{0} \exp \left(-\alpha_{\mathrm{rad}} z\right)$ is the modal field amplitude in the waveguide grating section at abscissa $z . \alpha_{\text {rad }}$ represents in a lossless waveguide the radiation loss due to all existing radiation orders into both the substrate and the cover. $\alpha_{\text {rad }}$ is the parameter that governs the whole coupling mechanism. The inverse of $\alpha_{\text {rad }}$ is the mode propagation length $L$, i.e., the length after which the propagating power falls by a factor $\mathrm{e}^{2}$.

Another feature of a coupling grating is the number of diffracted orders. Clean detection conditions and high coupling efficiency requirements lead to the general recommendation that the grating period $A$ must be small enough to only allow only one or very few propagating diffraction orders.

A last important feature of an actual coupling grating is that the grating is necessarily of finite area, and diffracts a beam of finile cross section. Such geometrical characteristics have to be carefully taken into account for the proper functioning of a practical grating coupler.

\section{The radiation coefficient}

In most realistic grating coupling situations, the grating length, $L=1 / \alpha_{\text {rad }}$, is of the order of the coupled beam cross section. This means in practice that $\alpha_{\text {rad }}$ must typically be of the order of 1 to $10 \mathrm{~cm}^{-1}$. It is well known that high-sensitivity evanescent-wave sensing of small species placed at a waveguide surface requires a waveguide of large guidance; this in turn implies that $\alpha_{\text {rad }}$ is a rapidly growing function of the grating depth. It is recognized that the typical depth $2 \sigma$ of the grating grooves made in $\mathrm{TiO}_{2}, \mathrm{Ta}_{2} \mathrm{O}_{5}, \mathrm{Si}_{3} \mathrm{~N}_{4}$ or $\mathrm{ZrO}_{2}$ waveguide films for the applications discussed here is of the order of 5 to $15 \mathrm{~nm}$, as can easily be checked in using Eq. (1) below. This means that the ratio between the depth and the period of the grating, $2 \sigma / \Lambda$, is very small; even in the case of a single diffracted order $\left(\Lambda<2 \lambda /\left(n_{\mathrm{e}}+n_{\mathrm{s}}\right)\right.$, where $n_{\mathrm{e}}$ is the mode effective index, $\lambda$ the wavelength), this ratio is at most 0.05 . This statement is very important for the design problematics of the grating coupling structures needed in this field: it means that the Rayleigh-Fourier method is well within its validity domain ( $\sigma / \Lambda$ should be smaller than $7 \%$ ) as shown by the yet unpublished results obtained after a systematic comparison with the exact methods of the Laboratoire d'Optique Electromagnétique of Marseille [10] in the framework of BRITE project BE-5720. Thus, all the rich and physically meaningful power of the Rayleigh-Fourier approach can be exploited to obtain analytical expressions and to achieve easily the synthesis of the desired optical functions. This is true in particular for the important parameter $\alpha_{\mathrm{rad}}$. In the case of a TE mode radiated in both substrate and cover, and for the -1 st diffraction order, $\alpha_{\text {rad }}$ takes the form

$$
\begin{aligned}
\alpha_{\mathrm{rad}}= & \left(\frac{k \sigma}{2}\right)^{2} \frac{n_{\mathrm{f}}^{2}-n_{\mathrm{c}}^{2}}{n_{\mathrm{e}} h_{\mathrm{e}}} \\
& \times \frac{\left(n_{\mathrm{f}}^{2}-n_{\mathrm{c}}^{2}\right)\left\{N_{\mathrm{s}} N_{\mathrm{f}}^{2}+N_{\mathrm{c}}\left[N_{\mathrm{s}}^{2}+\left(n_{\mathrm{f}}^{2}-n_{\mathrm{s}}^{2}\right) \cos ^{2} \psi\right]\right\}}{\left(N_{\mathrm{f}}^{2}+N_{\mathrm{s}} N_{\mathrm{c}}\right)^{2}-\left(n_{\mathrm{f}}^{2}-n_{\mathrm{s}}^{2}\right)\left(n_{\mathrm{f}}^{2}-n_{\mathrm{c}}^{2}\right) \cos ^{2} \psi}
\end{aligned}
$$

where $h_{\mathrm{e}}$ is the effective waveguide thickness and

$\psi=k N_{\mathrm{f}} h, N_{j}=\sqrt{n_{j}^{2}-\left(n_{\mathrm{e}}-\lambda / \Lambda\right)^{2}}, j=\mathrm{c}, \mathrm{f}, \mathrm{s}, k=2 \pi / \lambda$

Eq. (1) is the sum of two terms: the first one corresponds to the radiation into the substrate, and the second one to radiation into the cover. It was obtained for a sinusoidal corrugation [11] but it is still valid for the estimation of $\alpha_{\mathrm{rad}}$ in arbitrary shallow groove profiles if one uses for $\sigma$ the amplitude of the first harmonic in the Fourier development of the considered profile. A very similar expression is obtained for the radiation coefficient due to diffraction order $m$, in which case the $-\lambda / \Lambda$ term in the expression for $N_{j}$ is replaced by $+m \lambda / \Lambda$ and $\sigma$ is the amplitude of the $m$ th harmonic in the Fourier development of the groove profile. Eq. (1) reveals an oscillatory dependence of the radiation coefficient on the waveguide film thickness $h$. This is due to interference effects between radiated waves and waves reflected by the waveguide walls after being radiated. This effect is completely absent in graded-index waveguides but, in the present case of large guidance step-index waveguide $\left(\left(n_{\mathrm{f}}-n_{\mathrm{s}}\right)\right.$ and $\left(n_{\mathrm{f}}-n_{\mathrm{c}}\right)$ large $)$, it can have a dramatic influence on $\alpha_{\mathrm{rad}}$.

As an example, Eq. (1) was used to calculate $\alpha_{\text {rad }}$ in the case of a $\mathrm{TiO}_{2}$ waveguide with $n_{\mathrm{f}}=2.4$, deposited on a silica substrate, $n_{\mathrm{s}}=1.46$, with a water-based cover, $n_{\mathfrak{c}}=1.33$, at 
the HeNe wavelength of $633 \mathrm{~nm}$. The grating period is 0.4 $\mu \mathrm{m}$, which corresponds to the case where only the -1 st order exists. This structure was optimized for a maximum sensitivity in the differential detection scheme using the $\mathrm{TE}_{0}$ and $\mathrm{TM}_{0}$ modes [12]. This leads to a waveguide depth $h=140$ $\mathrm{nm}$. The grating is at the guide-cover interface. The dependence of the radiation coefficient on the half-depth $\sigma$ of the grooves is in such a case given by

$\alpha_{\mathrm{rad}}^{\mathrm{TE}}=\left(\frac{k \sigma}{2}\right)^{2} \times 4.20 \mathrm{~cm}^{-1}$

$\alpha_{\mathrm{rad}}^{\mathrm{TM}}=\left(\frac{k \sigma}{2}\right)^{2} \times 3.97 \mathrm{~cm}^{-1}$

where the radiation coefficient of the TM mode is given by an analytical expression, too intricate to be given here, which can be found in Ref. [11]. Using Eqs. (2) gives the required grating depth. For instance, $\alpha_{\text {rad }}=5 \mathrm{~cm}^{-1}$ corresponds to $\sigma=4.40 \mathrm{~nm}$ for the TE mode and to $\sigma=4.52 \mathrm{~nm}$ for the TM mode.

It is important to note that the previous expressions and estimations are valid for a single-undulation grating at the guide-cover interface. In the case of two corrugated waveguide boundaries, the situation is completely changed, since the total diffraction product depends on the interference between the diffraction products of both undulations and also on the reflections at the waveguide boundaries. One finds for a TE mode the following analytical result:

$$
\begin{aligned}
\alpha_{\mathrm{rad}}^{\text {total }}= & \alpha_{\mathrm{c}}+\alpha_{\mathrm{s}} \\
\alpha_{\mathrm{c}}= & \frac{N_{\mathrm{c}}\left(n_{\mathrm{f}}^{2}-n_{\mathrm{e}}^{2}\right)}{n_{\mathrm{e}} h_{\mathrm{e}} G}\left\{S_{\mathrm{c}}^{2}\left[N_{\mathrm{c}}^{2}+\left(n_{\mathrm{f}}^{2}-n_{\mathrm{c}}^{2}\right) \cos ^{2} \psi\right]\right. \\
& \left.-2 S_{\mathrm{c}} S_{\mathrm{s}} N_{\mathrm{f}}^{2} \cos \psi+S_{\mathrm{s}}^{2} N_{\mathrm{f}}^{2}\right\} \\
\alpha_{\mathrm{s}}= & \frac{N_{\mathrm{s}}\left(n_{\mathrm{f}}^{2}-n_{\mathrm{e}}^{2}\right)}{n_{\mathrm{e}} h_{\mathrm{e}} G}\left\{S_{\mathrm{c}}^{2} N_{\mathrm{f}}^{2}-2 S_{\mathrm{c}} S_{\mathrm{s}} N_{\mathrm{f}}^{2} \cos \psi\right. \\
& \left.+S_{\mathrm{s}}^{2}\left[N_{\mathrm{s}}^{2}+\left(n_{\mathrm{f}}^{2}-n_{\mathrm{s}}^{2}\right) \cos ^{2} \psi\right]\right\} \\
S_{j}= & \frac{k \sigma_{\mathrm{c}}}{2} \sqrt{n_{\mathrm{f}}^{2}-n_{j}^{2}}, \quad j=\mathrm{c}, \mathrm{s} \\
G= & \left(N_{\mathrm{f}}^{2}+N_{\mathrm{c}} N_{\mathrm{s}}\right)^{2}-\left(N_{\mathrm{f}}-N_{\mathrm{c}}\right)\left(N_{\mathrm{f}}-N_{\mathrm{s}}\right) \cos ^{2} \psi
\end{aligned}
$$

where $\sigma_{\mathrm{s}}$ and $\sigma_{\mathrm{c}}$ are the half-depths of the lower and upper undulations, respectively.

The results of Eqs. (1) and (3) and their differences are dramatically illustrated in Fig. 2, showing the radiation coefficient of TE and TM modes at either side of the normal radiation direction in the presence of a single radiation order (a second order appears in the neighbourhood of $+25^{\circ}$ radiation) in the waveguide designed for maximum TM/TE differential sensitivity corresponding to expressions (2). The amplitude of the single undulation, as well as $\sigma_{s}$ and $\sigma_{c}$, are $10 \mathrm{~nm}$. The variation of the radiation angle $\theta$ is obtained by varying the grating period. Whereas $\alpha_{\text {rad }}$ for both TE and TM

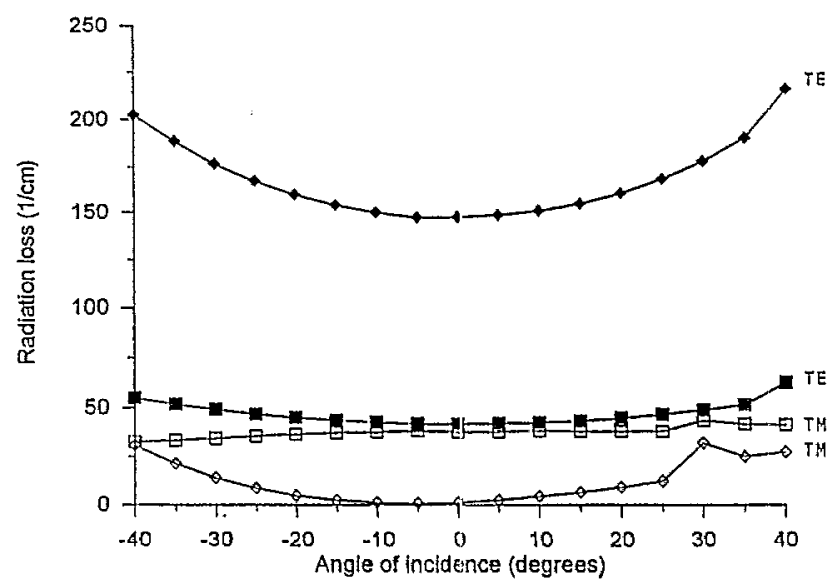

Fig. 2. Dependence of the total radiation coefficient $\alpha_{\mathrm{rad}}$ vs. the output angle $\theta$ in the substrate for single-sided $(\square)$ and double-sided $(\diamond)$ corrugations for TE (filled symbols) and TM (open symbols) guided modes. Data are those given as an example in the text.

modes with a single corrugation remain about the same, they differ considerably in the dual-undulation case: $\alpha_{\mathrm{Tad}}^{\mathrm{TE}}$ is roughly four times as large as the former, which reveals a constructive interference between the two diffraction products, and $\alpha_{\text {rad }}^{\mathrm{TM}}$ reaches practically zero at normal diffraction. This example should not be taken as illustrating a general behaviour; another waveguide thickness may well lead to a contrary situation. But it illustrates how carefully one should design a differential, dual-polarization evanescent-wave sensor [12] to make sure that both polarizations are excited.

Although the above expressions for $\alpha_{\mathrm{rad}}$ represent only the first step for the evaluation of the important in-coupling efficiency, they can readily be used for the design of out-coupling grating sensors [13], for instance, for the evaluation of the tolerances on the waveguide geometry, and to avoid the pifalls due to interference cffects of the waveguide walls.

\section{The input coupling efficiency}

The next most important set of parameters for a properly designed in-coupling grating is that of the geometrical matching between grating area and incident beam. In a uniform grating of infinite extent in the direction orthogonal to the plane of incidence, and semi-infinite extent in the direction opposed to the coupled mode propagation, the two essential parameters are the width $2 \mathrm{~W}$ of the incident beam, assumed here to be Gaussian, and the distance $X$ between the beam centre intersection in the grating plane and the front end of the grating area. It is advantageous to deal with these two parameters in a normalized fashion with respect to the total radiation coefficient or, if the structure has absorption and scattcring losses, with respect to the total attenuation coefficient. The conditions of maximum waveguide excitation effciency have long been known to be given by [14]

$$
\begin{aligned}
& \alpha_{\text {tot }} W=0.68 \cos \theta \\
& \alpha_{\text {tot }} X=0.5
\end{aligned}
$$


This is a result of the optimization of the guided-mode field at the edge of the grating area: this arises as an optimized balance between the field coupling into the waveguide, and its being re-radiated outside by the very same grating. $\alpha_{\text {tot }}$ is the total loss coefficient equal to the sum of the radiation coefficients of all propagating radiation orders, plus, in the case of lossy waveguide gratings, the absorption and scattering coefficients. Defining the coupling coefficient $\eta$ as the ratio between the coupled mode power at the end of the grating area to the incoming power propagated by the incident Gaussian beam, $\eta$ has an upper limit of $81 \%$ [12], which can be reached in the case where there is a single diffraction order in the substrate and no order in the cover. In all other cases, where there is also one order in the cover, or when there are more diffraction orders, satisfying the optimum conditions (4) will lead to a lower maximum excitation efficiency $\eta^{*}$, via order $i$ incident in medium $j$, given by

$\eta^{*}=0.81 \alpha_{\text {radj }}^{i} / \alpha_{\text {tot }}$

where $\alpha_{\operatorname{rad} j}{ }^{i}$ is the radiation coefficient into medium $j$ ( $j=\mathrm{s}$ or c) via diffraction order $i$. The evaluation of the maximum achievable in-coupling coefficient $\eta^{*}$ thus requires the knowledge of the radiation coefficient of the used order into the medium from which the excitation takes place. In the case of TE polarization, the radiation $\alpha_{\mathrm{rad} \mathrm{s}}^{-1}$ and $\alpha_{\mathrm{rad} \mathrm{c}}{ }^{-1}$ produced by the -1 st order of a grating placed at the waveguidecover interface is

$$
\begin{aligned}
\alpha_{\mathrm{radc}}^{-1}= & \left(\frac{k \sigma}{2}\right)^{2} \frac{n_{\mathrm{f}}^{2}-n_{\mathrm{e}}^{2}}{n_{\mathrm{e}} h_{\mathrm{e}}} \\
& \times \frac{\left(n_{\mathrm{f}}^{2}-n_{\mathrm{c}}^{2}\right) N_{\mathrm{c}}\left[N_{\mathrm{s}}^{2}+\left(n_{\mathrm{f}}^{2}-n_{\mathrm{s}}^{2}\right) \cos ^{2} \psi\right]}{\left(N_{\mathrm{f}}^{2}+N_{\mathrm{s}} N_{\mathrm{c}}\right)^{2}-\left(n_{\mathrm{f}}^{2}-n_{\mathrm{s}}^{2}\right)\left(n_{\mathrm{f}}^{2}-n_{\mathrm{c}}^{2}\right) \cos ^{2} \psi}
\end{aligned}
$$

$$
\begin{aligned}
\alpha_{\operatorname{rad~s}}^{-1}= & \left(\frac{k \sigma}{2}\right)^{2} \frac{n_{\mathrm{f}}^{2}-n_{\mathrm{s}}^{2}}{n_{\mathrm{e}} h_{\mathrm{e}}} \\
& \times \frac{\left(n_{\mathrm{f}}^{2}-n_{\mathrm{c}}^{2}\right) N_{\mathrm{s}} N_{\mathrm{f}}^{2}}{\left(N_{\mathrm{f}}^{2}+N_{\mathrm{s}} N_{\mathrm{c}}\right)^{2}-\left(n_{\mathrm{f}}^{2}-n_{\mathrm{s}}^{2}\right)\left(n_{\mathrm{f}}^{2}-n_{\mathrm{c}}^{2}\right) \cos ^{2} \psi}
\end{aligned}
$$

In practical situations, it may be interesting or simply necessary to focus the incident beam slightly by means of a lens onto the grating. The optimum excitation conditions become more complex. They will be reported elsewhere [15].

Expressions (4) to (6) give the conditions for coupling the maximum relative power into a waveguide at the end of the grating area. This pertains to sensor designs where the function of the grating is just to couple the power in and out. In cases where the grating also participates in the sensing, the requirements on the coupling efficiency are quite different: the incident beam impinges onto the central region of the grating area, therefore most of the power coupled in gets reradiated before reaching the edge of the grating area. The abscissa $X$ where the guided power reaches its maximum is

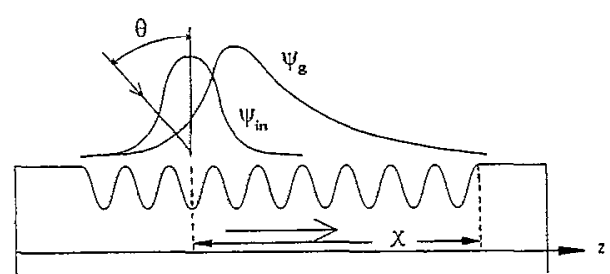

Fig. 3. Sketch of waveguide excitation from a Gaussian free-space wave field $\psi_{\mathrm{in}}$ under incidence $\theta$ by means of a finite-size grating when the distance $X$ between the incidence beam impact and the grating edge is large. The coupled field $\psi_{\mathrm{g}}$ reaches its maximum value within the grating area.

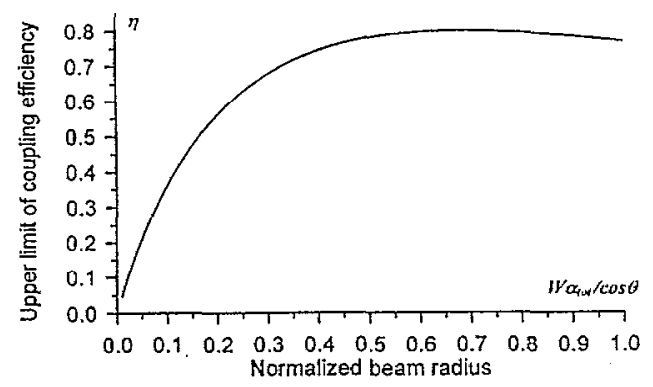

Fig. 4. Graph of the maximum coupling efficiency $\eta$ obtainable in a grating waveguide within the grating area when the incident beam impact is far from the grating front edge vs. the normalized incident beam radius $W \alpha_{\text {tot }} / \cos \theta$.

within the grating, as sketched in Fig. 3. Therefore assuming the edge of the grating area to be far away from the impact of the incident beam (say, further than $3 W / \cos \theta$ ), we can still find the excitation conditions for a maximum guided power (i.e., $81 \%$ ) within the grating area. This optimization problem concerns here a single normalized parameter: the normalized incident beam width $\alpha_{\mathrm{tot}} W / \cos \theta$.

Using the Rayleigh-Fourier method again leads to Fig. 4, giving the excitation efficiency versus the normalized beam size. This is the coupling efficiency that can actually be achieved in the case of a single diffraction order in the substrate. The way to use this normalized graph to obtain the coupling efficiency in any other structure is to give the incidence angle $\theta$, the total attenuation coefficient, and the radiation coefficient $\alpha_{\operatorname{rad} j}{ }_{j}^{i}$. With Fig. 4 giving $\eta$, the coupling efficiency $\eta^{*}$ via diffraction order $i$ from medium $j$ is given by

$\eta^{*}=\eta \alpha_{\mathrm{tad} j}^{i} / \alpha_{\mathrm{tot}}$

Assuming for instance $\alpha_{\mathrm{tot}}=5 \mathrm{~cm}^{-1}$ and a radiation coefficient into the substrate of $2.5 \mathrm{~cm}^{-1}$, a bcam diameter $2 W=0.3 \mathrm{~mm}$ and an incidence angle of $15^{\circ}$, the excitation efficiency is $12 \%$.

\section{Conclusions}

It has been shown how the Rayleigh-Fourier method can produce the physical concepts and analytical results allowing an intelligent and simple design of optical biochemical waveguide grating sensors. Analytical expressions are given allowing the full design of step-index waveguide grating 
sensors with one and two corrugated boundaries using a TE mode radiated or excited via the -1 st diffraction order in the recommended application case where the latter is the only propagating order. TM sensing as well as cases involving more than one diffraction order can be treated similarly using the results of the cited literature.

\section{Acknowledgements}

This work was performed under BRITE project 'FOA', BE-5720.

\section{References}

[1] W. Lukosz and K. Tiefenthaler, Directional switching in planar waveguides effected by adsorption-desorption processes, 2 nd Eur. Conf. Integrated Optics, Florence, Italy, 1983, IEE Conference Publication No. 227, London, 1983, pp. 152-155.

[2] A. Bernard and H.R. Bosshard, Real time monitoring of antigenantibody recognitionn on a metal oxide surface by an optical grating coupling sensor, Eur. J. Biochem., 230 (1995) 416.

[3] O. Parriaux and P. Sixt, Sensitivity optimization of grating coupled evanescent wave immunosensors, Sensors and Actuators B, 29 (1995) 289-292.

[4] O. Parriaux, V.A. Sychugov and A.V. Tishchenko, Coupling gratings as waveguide functional elements, J. Pure Appl. Opt., 5 (1996) 453469.
[5] Li Feng Li, Analysis of planar waveguide grating coupled with double surface corrugations of identical period, Opt. Commun., 114 (1995) $406-412$.

[6] N.F. Hartman, D. Campbell and M. Gross, Waveguide interferometer configurations, Proc. IEEE-LEOS' '88, Santa Clara, CA, USA, 1988, pp. 298-299.

[7] R.E. Kunz, J. Edilinger, P. Sixt and M.T. Gale, Replicated chirped waveguide grating for optical sensing applications, Sensors and Actuators A, 47 (1995) 482-486.

[8] K. Tiefenthaler and W. Lukosz, Grating couplers as integrated optical humidity and gas sensors, Thin Solid Films, 126 (1985) 205-211.

[9] R.E. Kunz, J. Edlinger, B.J. Curtis, M.T. Gale, L.U. Lempen, H. Rudigier and $\mathrm{H}$. Schütz, Grating couplers in tapered waveguides for integrated optical sensing, Proc. SPIE, Vol. 2068, 1994, pp. 3:3-325.

[10] M. Nevière, A.V. Tishchenko and V.A. Sychugov, Comparison between exact and Rayleigh-Fourier methods for the design of waveguide coupling gratings, unpublished work carried out under project BRITE BE-5720, 1995.

[11] V.A. Sychugov and A.V. Tishchenko, Light emission from corrugated dielectric waveguides, Sov. J. Quantum Electron., 10 (1980) 1175.

[12] Ch. Fattinger, Integrated optic biochemical sensors, Eur. Conf. Insegrated Optics, ECIO '95, Delft, Netherlands, 3-6 April, 1995, pp. $439-444$

[13] D. Clerc and W. Lukosz, Affinity and immunosensing with an integrated optical output-grating coupler, Abstract, EUROPT(R)ODE II, Florence, Italy, 19-21 April, 1994.

[14] T. Tamir, in T. Tamir (ed.), Integrated Optics, Vol. 7 of Topics in Applied Physics, Springer, Bcrlin, 1979, p. 105.

[15] N.M. Lyndin, O. Parriaux and V.A. Sychugov, Waveguide excitation by a Gaussian beam and a finite size grating, Sensors and Actuators $B$, submitted for publication. 